Journal of Computer Sciences 1(4): 515-520, 2005

ISSN 1549-3636

(C) 2005 Science Publications

\title{
Improved AIMD- A Mathematical Study
}

\author{
${ }^{1}$ V.S.P. Srivastav, ${ }^{2}$ M.K. Gupta, ${ }^{2}$ Sachin Kumar and ${ }^{3}$ Kadambri Agarwal \\ ${ }^{1}$ Computer Division, IGNOU, Maidan Garhi, New Delhi-110068, India \\ ${ }^{2}$ Department of Mathematics, CCS University, Meerut 250004, India \\ ${ }^{3}$ IIMT College, Meerut, India
}

\begin{abstract}
One of the crucial elements in the Internet is the ability to adequately control Congestion. AIMD (Additive Increase Multiplicative Decrease) is the best algorithm among the set of liner algorithms because it reflects good efficiency as well as good fairness. Our Control model is based on original approach of AIMD. In this paper we introduce improved version of AIMD. We call our approach improved AIMD. We are also including various inherent properties of Congestion Control i.e. Fairness, Responsiveness, Smoothness and efficiency.
\end{abstract}

Key words: Congestion control, TCP friendly, liner algorithms, RTTs

\section{INTRODUCTION}

Congestion Control in the Internet was introduced in the late 1980s by Van Jacobson ${ }^{[1]}$. A network is considered congested when too many packets try to access the same route, resulting in an amount of packets being dropped. In this state, the total load exceeds the capacity of the network. During congestion, actions are taken both by transmission protocols and network router in order to avoid a congestive collapse ensure network stability, efficiency and fair resources allocation of bandwidth. During a time of collapse, only a fraction of bandwidth is utilized and remaining is wasted.

In the last few years, many congestion control algorithms have been introduced ${ }^{[1-4]}$. Since the dominant Internet flow is TCP based ${ }^{[5]}$, it is widely accepted that new algorithm should be TCP friendly. A System is said to be TCP friendly if Non TCP and TCP flow have approximately the same data-transferring rate (in terms of packets per second) under same conditions ${ }^{[6,7]}$. The following are the basic properties of congestion control protocol.

Efficiency: It is the average flows throughput per round trip time (RTT) when system is in equilibrium. System is said to be in equilibrium when each flow shares same window.

Smoothness: It is magnitude of oscillations during decrease step ${ }^{[8]}$.

Responsiveness: It is number of RTTs required for the system to achieve equilibrium ${ }^{[8]}$.

Fairness: Every flow uses equal share of bandwidth.
There are a number of linear algorithms introduced till now. In linear algorithm increasing factor and decreasing factor varies linearly. e.g. AIMD $^{[8]}$ (Additive increase/ Multiplicative decrease) $\mathrm{MIMD}^{[9]}$ (Multiplicative increase/ Multiplicative decrease), MIAD $^{[9]}$ (Multiplicative Increase/ Additive Decrease) and $\mathrm{AIAD}^{[9]}$ (Additive Increase/ Additive Decrease). But long-term fairness is achieved by AIMD $^{[9]}$. Our proposed work is related to AIMD family wherein we present an improvement of AIMD algorithms that improves fairness as well as efficiency.

AIMD congestion control basic technique and system model: Chiu and Jain provide a theoretical justification for favoring $\operatorname{AIMD}^{[3]}$ : according to their analysis of linear adjustment algorithm for a simple feedback model, AIMD yields the quickest Convergence to efficiency -fair states ${ }^{[9]}$.

Within the class of increase decrease method; we specifically focus on the class of Additive Increase and Multiplicative Decrease (AIMD). In AIMD when system responds to congestion, used Bandwidth (Window) is multiplied by some factor (Decrease step) and in the absence of Congestion used Window is increased by some factor (Increase Step). Suppose these factors are $\mathrm{a}$ and $\mathrm{b}$ respectively. Many researchers have proved $a=1$ and $b=\frac{1}{2}$ for best utilization of channel. Obviously we follow these factors. But in our proposed work these are implemented in such a way that system gives better efficiency than that of previous works.

Our system is binary and synchronized. System is synchronized because every user has same RTT and the system gives feedback simultaneously for each user. The system feedback is 1 when window is available. Our system model is defined in following figure that is based on assumption of Chiu and Jain model ${ }^{[3]}$. 


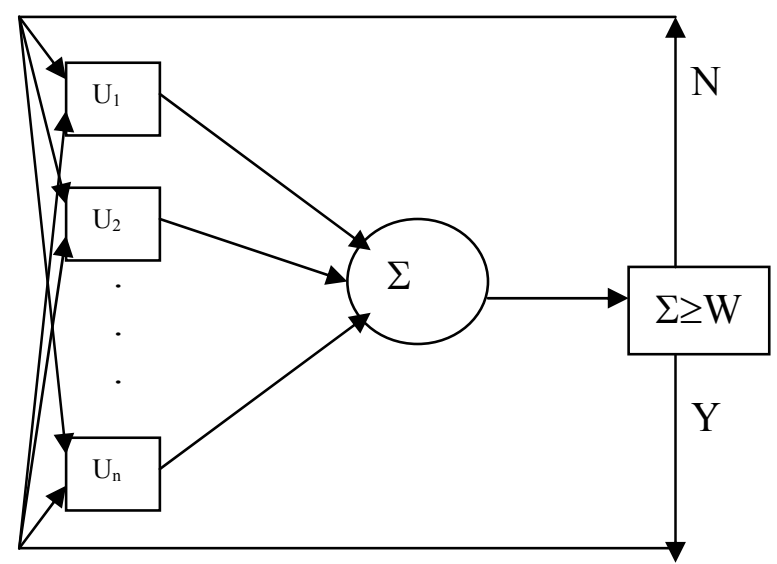

The system has $n$ users $U_{1}, U_{2}, U_{3} \ldots U_{n}$ and shares $W_{1}, W_{2}, W_{3} \ldots W_{n}$ windows and network window size is given by $W$. If there is congestion, system gives 0 response otherwise system gives 1 . If feedback is 1 , then $a=1$ is increased in all user windows, if feedback is 0 then $b=\frac{1}{2}$ is multiplied in all users window.

A pseudocode of improved AIMD: Let us assume network capacity (Window size) is $W$. For Simplicity let us assume we have two flows system $\mathrm{f} 1$ and $\mathrm{f} 2$. Initially let flows $\mathrm{f} 1$ and $\mathrm{f} 2$ contain $x$ and $y$ window respectively. With out loss of generality we assume that $x<y$ and $x+y<W$ furthermore, we are assuming that system converges to 'fair' in ' $m$ ' cycle. In $1^{\text {st }}$ cycle Pseudocode is given by:

Flow f1

Flow f2

$x$

$x+1$

$y$

$x+1+1$

$y+1$

$x+1+1$

$y+1+1$

$x+1+1 \ldots k_{1}$ times

$$
y+1+1 \ldots k_{1} \text { times }
$$

It gives

$x+k_{1}$

$$
y+k_{1}
$$

Thus total flow is $x+y+2 k_{1}$

It is clear in $1^{\text {st }}$ cycle that system has $k_{1}+1$ Round Trip Time (RTTs) or steps. Let $x+y+2 k_{1} \geq W$ then there is Congestion and system gives 0 feedback. Now we will use decrease step. In $2^{\text {nd }}$ cycle Pseudocode is given by:
Flow f1

$\frac{x}{2}+k_{1}$

$\frac{x}{2}+k_{1}+1$

$\frac{x}{2}+k_{1}+1+1$

.

$\frac{x}{2}+k_{1}+1+1 \ldots k_{2}$ times

$\frac{y}{2}+k_{1} \ldots k_{2}$ times

It gives

$\frac{x}{2}+k_{1}+k_{2} \quad \frac{y}{2}+k_{1}+k_{2}$

Thus total flow is $\frac{x}{2}+\frac{y}{2}+2 k_{1}+2 k_{2}$.

Obviously $2^{\text {nd }}$ cycle contains $k_{2}+1$ RTT. Let $\frac{x}{2}+\frac{y}{2}+2 k_{1}+2 k_{2} \geq W$ then system gives ' 0 ' feedback. Obviously we will use decrease step. In $3^{\text {rd }}$ cycle Pseudocode is given by:

Flow f1

Flow f2

$\frac{x}{2^{2}}+k_{1}+k_{2}$

$\frac{y}{2^{2}}+k_{1}+k_{2}$

$\frac{x}{2^{2}}+k_{1}+k_{2}+1$

$\frac{y}{2^{2}}+k_{1}+k_{2}+1$

$\frac{x}{2^{2}}+k_{1}+k_{2}+1+1$

$\frac{y}{2^{2}}+k_{1}+k_{2}$

$\frac{x}{2^{2}}+k_{1}+k_{2} \ldots k_{3}$ times $\frac{y}{2^{2}}+k_{1}+k_{2} \ldots k_{3}$

times.

It gives

$\frac{x}{2^{2}}+k_{1}+k_{2}+k_{3} \quad \frac{y}{2^{2}}+k_{1}+k_{2}+k_{3}$

Thus total flow is $\frac{x}{2^{2}}+\frac{y}{2^{2}}+2 k_{1}+2 k_{2}+2 k_{3}$. 
Here $3^{\text {rd }}$ cycle contains $k_{3}+1$ RTTs. Let $=\left(k_{1}+1\right)(x+y)+k_{1}\left(k_{1}+1\right)$

$\frac{x}{2^{2}}+\frac{y}{2^{2}}+2 k_{1}+2 k_{2}+2 k_{3} \geq W$ then system gives $0=\left(k_{1}+1\right)\left(x+y+2 k_{1}\right)$

feedback. Obviously we will use decrease step. Similarly at $\mathrm{m}^{\text {th }}$ cycle we have:

Flow f1

Flow f2

$$
\begin{aligned}
& \frac{x}{2^{m-1}}+k_{1}+k_{2} \ldots k_{m} \\
& \frac{y}{2^{m-1}}+k_{1}+k_{2} \ldots k_{m}
\end{aligned}
$$

Thus total flow is $\frac{x}{2^{m-1}} \frac{y}{2^{m-1}}+2 k_{1}+2 k_{2} \ldots 2 k_{m}$. Suppose $m^{\text {th }}$ cycle points to equilibrium that is all flows share fair allocation of resources.

The algorithmic approach when initial window size of 2 flows and Window size are $x, y$ and $W$ respectively, is given by:

$\operatorname{AIMD}(x, y, W)$

\{

$z=x+y / / z$ denotes used Capacity of Network.

$k=1, t=1 / / k$ denotes numbers of RTTs

while (1)

\{

$k=k+1$

$z=x+y+2 t$

$t=t+1$

if $(z>=W)$

\{

$x=\frac{x}{2}$

$y=\frac{y}{2}$

$z=x+y+2 t$

$k=k+1$

\}

\}

Total number of packets in various cycles: In $1^{\text {st }}$ Cycle, total number of packets is given by:

$x+(x+1)+(x+2) \ldots\left(x+k_{1}\right)+$

$y+(y+1)+(y+2) \ldots\left(y+k_{1}\right)$

$=\left(k_{1}+1\right)(x+y)+2\left(1+2+3 \ldots k_{1}\right)$

$=\left(k_{1}+1\right)(x+y)+2 k_{1}\left(\frac{k_{1}+1}{2}\right)$

But from Cycle $1^{\text {st }}$ we have $x+y+2 k_{1}=W$

Therefore $x+y+k_{1}=W-k_{1}$

Thus total numbers of packet is given by $\left(1+k_{1}\right)\left(W-k_{1}\right)$

In $2^{\text {nd }}$ Cycle, total number of packets is given by:

$\left(\frac{x}{2}+k_{1}\right)+\left(\frac{x}{2}+k_{1}+1\right)+\left(\frac{x}{2}+k_{1}+1+1\right) \ldots+\left(\frac{x}{2}+k_{1}+\right.$

$\left.k_{2}\right)+\left(\frac{y}{2}+k_{1}\right)+\left(\frac{y}{2}+k_{1}+1\right)+\left(\frac{y}{2}+k_{1}+1+1\right) \ldots+$

$\left(\frac{y}{2}+k_{1}+\mathrm{k}_{2}\right)$

After solving the equation we have:

$\left(1+k_{2}\right)\left(\frac{x}{2}+\frac{y}{2}+2 k_{1}+k_{2}\right)$

But from $2^{\text {nd }}$ cycle we have

$\left(\frac{x}{2}+\frac{y}{2}+2 k_{1}+2 k_{2}\right)=W$

Therefore $\frac{x}{2}+\frac{y}{2}+2 k_{1}+k_{2}=\mathrm{W}-\mathrm{k}_{2}$

Thus total number of packets is given by : $\left(1+k_{2}\right)\left(W-k_{2}\right)$

Similarly in $3^{\text {rd }}$ cycle, total number of packets is given by:

$\left(1+k_{3}\right)\left(W-k_{3}\right)$

Similarly $\mathrm{m}^{\text {th }}$ cycle, total number of packets is given by:

$\left(1+k_{m}\right)\left(W-k_{m}\right)$

Thus total number of packets in all cycles is given by:

$\left(1+k_{1}\right)\left(W-k_{1}\right)+\left(1+k_{2}\right)\left(W-k_{2}\right)+$

$\left(1+k_{3}\right)\left(W-k_{3}\right)+\ldots\left(1+k_{m}\right)\left(W-k_{m}\right)$

Relationship between RTTs in various cycles. From equation 1 and 2 we have

$x+y+2 k_{1}=\frac{x}{2}+\frac{y}{2}+2 k_{1}+2 k_{2}$

$k_{2}=\frac{1}{4}(x+y)$

But from equation 1 we have:

$x+y+2 k_{1}=W$ 


$$
\begin{aligned}
& k_{1}=(W-x-y) / 2 \\
& 4 k_{2}+2 k_{1}=W \\
& k_{2}=\left(W-2 K_{1}\right) / 4
\end{aligned}
$$

From equations 2 and 3 we have:

$$
\begin{aligned}
& \frac{x}{2}+\frac{y}{2}+2 k_{1}+2 k_{2}=\frac{x}{2^{2}}+\frac{y}{2^{2}}+2 k_{1}+2 k_{2}+2 k_{3} \\
& k_{3}=\frac{1}{8}(x+y) \\
& k_{3}=\frac{1}{2} k_{2}
\end{aligned}
$$

From equations 3 and 4 we have:

$$
\begin{aligned}
& k_{4}=\frac{1}{2} k_{3} \\
& k_{4}=\left(\frac{1}{2^{2}}\right) k_{2}
\end{aligned}
$$

Thus $k_{m}=\left(\frac{1}{2^{m-2}}\right) k_{2}$ for $m=3$

Analysis: In the analysis we specify basic factors of Congestion Control such as fairness, efficiency, responsiveness and smoothness respectively.

Fairness: One of the interesting properties of AIMD algorithm that we introduce in this paper is the ability of a scheme to approach to fairness monotonically, i.e. the fairness during interval ' $i$ ' is given by $f_{i}=\frac{x_{i}}{y_{i}}$, $0<=f_{i}<=1$, then the following conditions should be satisfied.

$$
\forall i: f_{i}+1>=f_{i} \text { and } \lim _{i \rightarrow \infty} f_{i}=1
$$

Without loss of generality we are assuming that $y=x+n$. At the end of $1^{\text {st }}$ cycle, fairness ratio is given by:

$$
\begin{aligned}
& \frac{\left(x+k_{1}\right)}{\left(y+k_{1}\right)}=\frac{\left(x+k_{1}\right)}{\left(x+n+k_{1}\right)} \\
= & 1-\frac{n}{\left(x+n+k_{1}\right)}
\end{aligned}
$$

Similarly at the end $2^{\text {nd }}$ cycle, fairness ratio is given by $1-\frac{n}{2}\left(\frac{1}{\left(\frac{x}{2}+\frac{n}{2}+k_{1}+k_{2}\right)}\right)$. Clearly term $\frac{n}{2}\left(\frac{1}{\left(\frac{x}{2}+\frac{n}{2}+k_{1}+k_{2}\right)}\right)$ is smaller than $\frac{n}{\left(x+n+k_{1}\right)}$. Similarly we can find fairness ratio for remaining cycle.

According to these results we can say that our system converge to monotonic fairness. There is one interested question here how much cycles are required for fairness. We have following reasoning for it.

Since every time both $\mathrm{x}$ and $\mathrm{y}$ are divided by 2 of its previous value and equal constant are added in both flows. Thus system can never reach equilibrium if we assume float arithmetic. In Integer arithmetic we are assuming that system reaches fairness in $\mathrm{m}$ cycle. It indicates that

$$
\begin{aligned}
& \frac{y}{2^{m-1}}+k_{1}+k_{2} \ldots k_{m}-\frac{x}{2^{m-1}}+k_{1}+k_{2} \ldots k_{m} \approx 1 \\
& \frac{y}{2^{m-1}}+\frac{x}{2^{m-1}} \approx 1 \frac{x}{2^{m-1}}+\frac{n}{2^{m-1}}-\frac{x}{2^{m-1}} \approx 1 \\
& n \approx 2^{m-1} \quad m \approx 1+\log (n)
\end{aligned}
$$

But in AIMD fairness is reflected as $1+\log (y)^{[10]}$.

Obviously Convergence to fairness of Improved AIMD is faster than that of AIMD.

Responsiveness: Numbers of RTTs required for equilibrium (Responsiveness) is measured as:

$$
\begin{aligned}
& \left(1+k_{1}\right)+\left(1+k_{2}\right)+\left(1+k_{3}\right) \ldots\left(1+k_{m}\right) \\
& =m+\left(k_{1}+k_{2}+k_{3} \ldots k_{m}\right) \\
& =m+\left(k_{1}+\left(\frac{w-2 k_{1}}{2}\right)\left(1-\left(\frac{1}{2}\right)^{m-1}\right)\right.
\end{aligned}
$$

In AIMD algorithm $k$ is defined as $k_{i}=\frac{W}{4}$ for $i>=2$.

It means number of RTTs is fixed in each cycle i.e. $\frac{W}{4}$ for $i>=2$. But in our approach $k_{i}=\frac{k_{i-1}}{2}$ for $i>=3$.It means number of RTTs in each cycle are half of its previous cycle for $i>=3$. Obviously we have less number of RTTs.

Smoothness: Smoothness is reflected between $i$ and $i+1$ cycle as:

$\frac{x}{2^{i-1}}+\frac{y}{2^{i-1}}+2 k_{1}+2 k_{2}+\ldots 2 k_{i}-\left(\frac{x}{2^{i}}+\frac{y}{2^{i}}+2 k_{1}+2 k_{2}+\ldots 2 k_{i}\right)$

$=\frac{x}{2^{i}}+\frac{y}{2^{i}}=\frac{x+y}{2^{i}}$

Where $\left(\frac{x}{2^{i}}+\frac{y}{2^{i}}+2 k_{1}+2 k_{2}+\ldots 2 k_{i}\right)$ is number of packets at the end of $i^{\text {th }}$ cycle and 
$\left(\frac{x}{2^{i}}+\frac{y}{2^{i}}+2 k_{1}+2 k_{2}+\ldots 2 k_{i}\right)$ is number of packets at the beginning of $(i+1)^{\text {th }}$ cycle. System becomes smoother if $i$ is increased.

It indicates that if numbers of cycle/RTTs (Responsiveness) are more, then smoothness becomes less. If Responsiveness is less then smoothness becomes more. This will be clearer from following example.

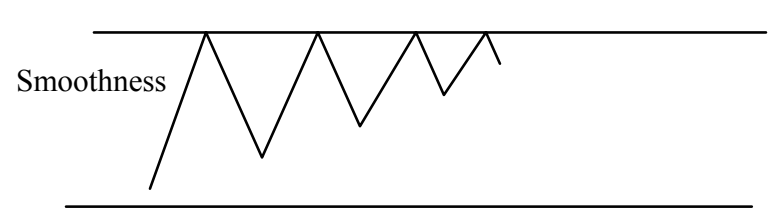

Responsiveness

Figure between responsiveness and smoothness

Efficiency: The average efficiency is an interesting and important property of a Congestion Control system. It is desired that the system achieve higher efficiency. First of all we develop an expression for average efficiency of all cycles i.e. $1^{\text {st }}$ cycle to equilibrium cycle. We know that total numbers of packets in $1^{\text {st }}$ cycle are $\left(1+k_{1}\right)\left(W-k_{1}\right)$. Since we have $\left(1+k_{1}\right)$ RTTs. Now we are interested the total numbers of packets in all $\mathrm{m}$ cycles. This is measured as:

$=\left(1+k_{1}\right)\left(W-k_{1}\right)+\left(1+k_{2}\right)\left(W-k_{2}\right)+\ldots$

$\left(1+k_{m}\right)\left(W-k_{m}\right)$

$=m W+(W-1)\left(k_{1}+k_{2}+\ldots k_{m}\right)-$

$\left(k_{1}^{2}+k_{2}^{2}+\ldots k_{m}^{2}\right)$

Solving this equation in term of $k_{1}$, we have:

$={ }_{m W+(W-1)}\left(k 1+\frac{W-2 k 1}{2}\right)\left(1-\left(\frac{1}{2}\right)^{m-1}\right)-\left(k_{1}^{2}+\frac{1}{12}(W-2 k 1)^{2}\left(1-\left(\frac{1}{4}\right)^{m-1}\right)\right)$

Thus average throughput in all $\mathrm{m}$ cycle can be achieved dividing above equation by

$W\left(k_{1}+k_{2}+\ldots k_{m}\right)$ We have:

$\left(\frac{m W+(W-1)\left(k 1+\frac{W-2 k 1}{2}\right)\left(1-\left(\frac{1}{2}\right)^{m-1}\right)-\left(k_{1}^{2}+\frac{1}{12}(W-2 k 1)^{2}\left(1-\left(\frac{1}{4}\right)^{m-1}\right)\right)}{W\left(k_{1}+k_{2}+\ldots k_{m}\right)}\right)$

$\left(\frac{m W+(W-1)\left(k 1+\frac{W-2 k 1}{2}\right)\left(1-\left(\frac{1}{2}\right)^{m-1}\right)-\left(k_{1}^{2}+\frac{1}{12}(W-2 k 1)^{2}\left(1-\left(\frac{1}{4}\right)^{m-1}\right)\right)}{W\left(k 1+\frac{W-2 k 1}{2}\right)\left(1-\left(\frac{1}{2}\right)^{m-1}\right)}\right)$
Average throughput in the equilibrium cycle (efficiency) is given by:

$$
\begin{aligned}
& \frac{\left(1+k_{m}\right)\left(W-k_{m}\right)}{W\left(1+k_{m}\right)} \\
= & \frac{1-k_{m}}{W} \\
= & \frac{1-k_{2}}{2^{m-2} W} \\
= & 1-\left(\frac{W-2 k_{1}}{4}\right)\left(\frac{1}{2^{m-2} W}\right)
\end{aligned}
$$

Example: Let the Network have $W=500$ and two users with initial loads of $x=10$ and $y=140$

Solution: Efficiency is given by $1-\left(\frac{W-2 k_{1}}{4}\right)\left(\frac{1}{2^{m-2} W}\right)$

Given $W=500, x=10$ and $y=140$

$k_{1}=\frac{W-x-y}{2}=175$

$m=1+\log (n))=1+\log \quad(y-x)=8 \quad$ (Integer

Arithmetic)

Efficiency $=1-\left(\frac{150}{4}\right)\left(\frac{1}{64 * 500}\right)$

$=.9989$ or $99.89 \%$

\section{CONCLUSION AND FUTURE WORK}

In this paper we presented and evaluated a new algorithm of AIMD family of congestion management, called Improved AIMD. It generalizes during increasing step $x=x+k$ and on decreasing step $\mathrm{x}=\mathrm{x}+\mathrm{k} / 2$. It converges to fairness in $1+\log$ (n) approximately. This is the best result in AIMD family. Responsiveness is reflected as very good because $k_{i}=\frac{k_{i-1}}{2}$ for $i>=3$. It gives smoothness $\frac{x+y}{2^{i}}$. Furthermore efficiency in equilibrium cycle is given by $1-\left(\frac{W-2 k_{1}}{4}\right)\left(\frac{1}{2^{m-2} W}\right)$. From above numerical figure it gives more than $99 \%$ efficiency. It is compare to the improved ${ }^{[10]}$. The issue that we have not included is the impact of different arrival time of each flow. It means that any flow can join the Network at any time. But in our work we assumed that arrival time of each flow is same. We will consider different arrival issue for future study. 


\section{REFERENCES}

1. Jacobson, V., 1988. Congestion avoidance and control. Proc. ACM SIGCOMM'88.

2. Floyd, S., M. Handley, J. Padhye and J. Widmer, 2000. Equation-based congestion control for unicast applications. Proc. ACM SIGCOMM 2000.

3. Chiu, D.M. and R. Jain, 1989. Analysis of the increase and decrease algorithms for congestion avoidance in computer neworks. Computer Networks and ISDN Systems, 17: 1-14.

4. Floyd, S., M. Handley and J. Padhye, 2000. Comparison of equation-based congestion control and AIMD-based congestion control. Work-inprogress. Available from http://www.aciri.org/tfrc.

5. Tan, W.T. and A. Zakhor, 1999. Real time Internet video using error resilient scalable compression and TCP-friendly transport protocol. IEEE Trans. Multimedia, 1: 172-186.
6. Mahadavi, J. and S. Floyd, 1997. TCP-friendly unicast rate-based flow control. Note sent to the end2end-interest mailing list.

7. Barden, B., D. Clark, J. Crowcroft, B. Davie, S. Deering, D. Estrin, S. Floyd, V. Jacobson, G. Minshall, C. Partridge, L. Peterson, K. Ramakrishnan, S. Shenker, J. Wroclawski and L. Zhang, 1998. Recommendations on queue management and congestion avoidance in the Internet. RFC 2309.

8. Flyod, S. and K. Fall, 1999. Promoting the use of end- to-end congestion control in the Internet. IEEE/ACM Trans. Networking, : 458-472.

9. Bansal, D. and H. Balakrishan, 2001. Binomial congestion control algorithms. Proc. IEEE INFOCOM., pp: 631-640.

10. Lahans, A. and V. Tsaoussidis, 2003. Exploiting the efficiency and fairness potential of AIMDbased congestion avoidance and control. Computer Networks, 43: 227-245. 